\title{
Quantifying the abnormal strain state in ferroelastic materials: a moment invariant approach
}

\author{
Lily Nguyen ${ }^{\mathrm{a}}$, Dong Wang ${ }^{\mathrm{b}}$, Yunzhi Wang ${ }^{\mathrm{b}, \mathrm{c}}$, Marc De Graef ${ }^{\mathrm{a}}$ \\ ${ }^{a}$ Department of Materials Science and Engineering, Carnegie Mellon University, 5000 Forbes \\ Avenue, Pittsburgh PA 15213, USA \\ ${ }^{b}$ Center of microstructure science, Frontier Institute of Science and Technology, State Key \\ Laboratory for Mechanical Behavior of Materials, Xi'an Jiaotong University, Xi'an 710049, \\ China \\ ${ }^{c}$ Department of Materials Science and Engineering, The Ohio State University, 2041 N. College \\ Rd. Columbus, $\mathrm{OH} 43210$
}

\begin{abstract}
The strain glass transition has been found in many ferroelastic systems, but the microstructural nature of strain glass is still unclear. Here, two-dimensional second and fourth order moment invariants as well as image entropy are used to evaluate the presence of a strain-glass state in simulated microstructure images of a doped ferroelastic system. Four different microstructural states are identified, depending on the doping concentration of point defects and the temperature. The martensitic state is characterized by a broad moment invariant distribution peaking near the invariants for the circle, whereas systems that display the strain glass transition produce distinctly different distributions. The image entropy is found to increase with increasing defect concentration; above the critical defect concentration of 0.1 , image entropy becomes nearly linearly dependent on temperature. The image analysis approach is capable of characterizing the range of strain domain shapes that occur in the different microstructural states of a doped ferroelastic system. Keywords: martensitic transformation; microstructure evolution; nanodomains; moment invariants; image entropy
\end{abstract}

Preprint submitted to Acta Materialia

May 6, 2015 


\section{Introduction}

The class of ferroelastic materials, which encompasses most shape memory alloys (SMAs) and superelastic materials, is an important class of functional materials and plays a crucial role in many modern technologies. These materials are defined by the non-linear strain-stress hysteresis and generally characterized by the existence of two distinct strain states, the strain liquid (a strain-disordered paraelastic state known as the parent phase or austenite at high temperature) and the strain crystal (a long-range strain-ordered ferroelastic state known as martensite at low temperature). The ferroelastic (or martensitic) transition between these two states under the influence of a temperature change or an applied stress gives rise to the technologically important shape memory effect and superelasticity $[1,2]$.

Recent experimental work [3-5] and simulations [6-8] have found that point defect doping will destroy the normal ferroelastic transition and produce a new strain state, a strain glass state, that is analogous to cluster-spin glasses in ferromagnetic systems and relaxors in ferroelectric systems [9]. Strain glass has shown abnormal characteristics, such as nanosized ferroelastic domains, frequency dispersion of the modulus and internal friction, a continuous phase transition and so on $[4,10-13]$. However, the physical nature of the strain glass state, i.e., the correlation between nanosized ferroelastic domains and long range ordered martensitic domains, as well as the correlation among different nanosized ferroelastic domains, is still unclear. A strain glass is a frozen and disordered strain state with short range order [3] due to point defects causing random local stresses and strains that inhibit the long-range ordering of strains [9]. Some of the properties of strain glass make it an interesting candidate for possible technological applica- 
tions; these include a "smeared" elastic modulus peak with frequency dispersion, the disappearance of a heat flow peak, and a small thermal hysteresis [11].

The strain glass state was first reported in $\mathrm{Ti}_{48.5} \mathrm{Ni}_{51.5}$ [3]; later Zhang et al. [14] built a detailed phase transition phase diagram and reported that the martensitic alloy $\mathrm{Ti}_{50-x} \mathrm{Ni}_{50+x}$ exhibits the strain glass state when doped with $x>1.3$ excess $\mathrm{Ni}$ as a point defect [3]. Several other alloy systems also exhibit the strain glass state: $\mathrm{Ti}_{50} \mathrm{Ni}_{50-x} \mathrm{Fe}_{x}$ with a critical Fe doping level of $5<x_{c}<6$ [4] and combinations of elements with Ti such as TiNi-X, Ti-Pd-X, etc., where $X$ is an alloying element such as Fe, Cr, Mn, Co, V, etc. [9].

While martensitic microstructures have been studied extensively, the key microstructural features of a strain glass state is still poorly understood. The presence of point defects, such as excess $\mathrm{Ni}$ in a $\mathrm{Ti}_{50-x} \mathrm{Ni}_{50+x}$ alloy, causes a strain glass transition to occur when the excess concentration $x$ is above a critical value $x_{c}$ [14]. Doping at a defect concentration lower than the critical value $x_{c}$ changes the normal martensitic phase transition temperature; the system forms a premartensitic tweed, a precursor that has a quasi-dynamic disordered strain state and is identified by its cross-hatched appearance in electron diffraction contrast images [3]. At or above the critical defect concentration, the normal martensitic phase transition is suppressed and a strain glass state forms. This state is represented by a static disordered strain glass and is similar in appearance to the premartensitic tweed [14]. However, premartensitic tweed is distinct from the strain glass state [9].

Wang et al. $[8,11]$ implemented a phase field model based on the Landau theory of phase transitions to study the effect of point defects on a ferroelastic system. The model assumes that point defects change the thermodynamic stability of 
martensite globally and create local lattice distortions that break up the symmetry of the Landau potential $[8,11]$. However, the microstructural nature such as domain size, distribution, shape, etc. of the strain glass state are still unclear.

In the present paper, we apply moment invariants and image entropy concepts to analyze the microstructure images produced by the model in [8] for different dopant concentrations at different temperatures in order to quantitatively determine the correlation between nanosized ferroelastic domains and long range ordered ferroelastic domains and among different nanosized ferroelastic domains. Moment invariants show that the strain glass state has similar shapes to that of the parent phase, while the premartensitic tweed is characterized by a wider range of isotropic shapes and the martensite is characterized by a broad moment invariant distribution peaking near the moment invariants for the circle. We will show that analysis of moment invariants and microstructure image entropy allows for the different microstructural states to be distinguished from each other.

\section{Theoretical and Modeling Approach}

\subsection{Moment Invariants}

Moment invariants (MIs) were first used by $\mathrm{Hu}$ in the area of pattern recognition to automate classification of different alphanumeric characters [15]. Moment invariants are (non-linear) combinations of Cartesian moments that are invariant with respect to certain classes of coordinate transformations: similarity transformations and affine transformations [16]. Similarity transformations include translations, rotations, and isotropic scaling, while affine transformations include all

similarity transformations and additionally, shearing and anisotropic scaling. The 2D Cartesian moments of an object can be defined in terms of the indicator func- 
tion $D(x, y)$ of the object:

$$
\mu_{p q}=\iint \mathrm{d} x \mathrm{~d} y x^{p} y^{q} D(x, y)=\iint_{D} \mathrm{~d} x \mathrm{~d} y x^{p} y^{q}
$$

where the order of the moment, $n$, is equal to the sum of the exponents $p+q$; the indicator function $D(x, y)$ equals 1 inside the object and 0 outside. If the moments are computed with respect to the center-of-mass coordinates $\left(x_{c}, y_{c}\right)$, then they are referred to as central moments and indicated by an over-bar:

$$
\bar{\mu}_{p q}=\iint_{D} \mathrm{~d} x \mathrm{~d} y\left(x-x_{c}\right)^{p}\left(y-y_{c}\right)^{q} .
$$

Invariance with respect to geometrical transformations is a desirable property allowing for shape descriptors that are independent of the size, orientation, and origin of the selected reference frame. For 1D distributions, there is typically no need to combine moments into invariants, since there is only one single moment of each order; in increasing order, and starting at $n=0$, the $1 \mathrm{D}$ moments of a distribution are known as $\mu_{0}$ (area under the curve), $\mu_{1}$ (mean), $\mu_{2}$ (variance), $\mu_{3}$ (skewness), and $\mu_{4}$ (kurtosis). In 2D and higher dimensional spaces, however, there are infinite ways to select the reference frame, so that invariant moment combinations become important. Our earlier work [16] has shown that these invariants can be used to identify shape classes; for instance, all ellipses can be shown to have one of their second order moment invariants to be equal to $16 \pi^{2}$ (or 1 in normalized units, see next paragraph), thus uniquely establishing the class of elliptical shapes. For higher order moments, it is clear from the integral in Eq. (1) that the object regions furthest away from the origin will have the largest contributions to the moments, so that higher order moment invariants provide increasingly detailed information about the object's outer edge; this is similar to the 1D case, where it 
is well known that the tails of a distribution affect the kurtosis more strongly than any of the lower order moments.

In earlier work, two independent second order MIs have been identified [16]; setting the surface area of the object to $A=\mu_{00}$, the normalized second order moment invariants are given by:

$$
\begin{aligned}
\bar{\omega}_{1} & =\frac{A^{2}}{2 \pi\left(\bar{\mu}_{20}+\bar{\mu}_{02}\right)} ; \\
\bar{\omega}_{2} & =\frac{A^{4}}{16 \pi\left(\bar{\mu}_{20} \bar{\mu}_{02}+\bar{\mu}_{11}^{2}\right)} .
\end{aligned}
$$

The normalization factors are chosen such that all 2D shapes have invariants that satisfy $0 \leq \bar{\omega}_{i} \leq 1$. For the circle we have $\bar{\omega}_{1}=\bar{\omega}_{2}=1$, and all shapes with isotropic moments of inertia (e.g., square, equilateral triangle, ...) lie on the parabola $\bar{\omega}_{2}=\bar{\omega}_{1}^{2}$. When the MIs for a large number of objects are plotted on the unit square with axes $\bar{\omega}_{i}$, the resulting distribution is referred to as the second order moment invariant map (SOMIM), shown schematically in Figure 2(a) [17].

Higher order moment invariants can be derived from complex moments; the 2D independent fourth order moment invariants are [17]:

$$
\begin{aligned}
& \bar{\tau}_{1}=\frac{A^{3}}{3 \pi^{2}}\left[\bar{\mu}_{40}+2 \bar{\mu}_{22}+\bar{\mu}_{04}\right]^{-1} \\
& \bar{\tau}_{2}=\frac{A^{6}}{48 \pi^{4}}\left[\bar{\mu}_{40} \bar{\mu}_{04}-4 \bar{\mu}_{31} \bar{\mu}_{13}+3 \bar{\mu}_{22}^{-2}\right]^{-1} \\
& \bar{\tau}_{3}=\frac{A^{9}}{1728 \pi^{6}}\left[\bar{\mu}_{40} \bar{\mu}_{22} \bar{\mu}_{04}-\bar{\mu}_{40} \bar{\mu}_{31}^{2}+2 \bar{\mu}_{31} \bar{\mu}_{13} \bar{\mu}_{22}-\bar{\mu}_{22}^{3}\right]^{-1},
\end{aligned}
$$

where $\bar{\tau}_{1}$ is a similarity moment invariant, and $\bar{\tau}_{2}$ and $\bar{\tau}_{3}$ are affine moment invariants. To avoid higher dimensional drawings of MI distributions, we use a reduced dimension representation; the graphical representation of the average second order versus the average fourth order moment invariants is referred to as the projected 
moment invariant map (PMIM) and is shown schematically in Figure 2(b) [17]. For all SOMIM and PMIM plots in the remainder of this paper, we urge the reader to consult Fig. 2 for the axes labels and scales.

\subsection{Phase field model for strain glass materials}

For simplicity, we consider a single crystal undergoing a generic improper square to rectangle (2D) martensitic transformation [18]. The Landau free energy (2-4-6 potential) without considering the effect of point defects is described by Equation (8) and the global transition temperature effect (GTTE) and the local field effect (LFE) caused by defects are described by Equations (9) and (10), respectively $[8,19]$ :

$$
\begin{aligned}
f_{c h}\left(\eta_{1}, \eta_{2}\right)=\frac{1}{2} A_{1}\left(\eta_{1}^{2}(\mathbf{r})\right. & \left.+\eta_{2}^{2}(\mathbf{r})\right)-\frac{1}{4} A_{2}\left(\eta_{1}^{4}(\mathbf{r})+\eta_{2}^{4}(\mathbf{r})\right) \\
+ & \frac{1}{4} A_{3}\left(\eta_{1}^{2}(\mathbf{r})+\eta_{2}^{2}(\mathbf{r})\right)^{2}+\frac{1}{6} A_{4}\left(\eta_{1}^{2}(\mathbf{r})+\eta_{2}^{2}(\mathbf{r})\right)^{3} \\
A_{1}=A_{1}^{0}\left(T-T^{0}(c)\right), \text { and } T^{0}(c)=T^{00}+b \cdot c & \eta_{i, j=1,2 ; m=1,3,5}^{\text {local }}(\mathbf{r}) \eta_{j}^{m}(\mathbf{r}) .
\end{aligned}
$$

The total free energy also includes the non-local gradient terms and the coherency elastic strain energy $E_{e l}$ :

$$
F=\int \mathrm{d}^{2} r\left[\frac{1}{2} \beta\left(\nabla \eta_{1}\right)^{2}+\frac{1}{2} \beta\left(\nabla \eta_{2}\right)^{2}+f_{c h}\left(\eta_{1}, \eta_{2}\right)+f_{L}(\mathbf{r})\right]+E_{e l},
$$

where

$$
\begin{aligned}
E_{e l}=\frac{1}{2} c_{i j k l} \sum_{p=1}^{2} \sum_{q=1}^{2} & \varepsilon_{i j}^{00}(p) \varepsilon_{i j}^{00}(q) \int \eta_{p}^{2}(\mathbf{r}) \eta_{q}^{2}(\mathbf{r}) \mathrm{d}^{3} r \\
& -\frac{1}{2} \sum_{p=1}^{2} \sum_{q=1}^{2} \int \frac{\mathrm{d}^{2} k}{(2 \pi)^{2}} B_{p q}\left(\frac{\mathbf{k}}{k}\right)\left\{\eta_{p}^{2}(\mathbf{r})\right\}_{k}\left\{\eta_{q}^{2}(\mathbf{r})\right\}_{k}^{*} .
\end{aligned}
$$


In these equations, $\eta_{i}(\mathbf{r})(i=1,2)$ are the long range order (lro) parameters characterizing the martensitic phase; $\eta_{i}^{\text {local }}(\mathbf{r})(i=1,2)$ describes the local field caused by the static point defects; $b$ characterizes the GTTE strength; $c$ is a dimensionless average defect concentration measured in terms of area fraction in the simulations; $T^{00}$ is the critical transition temperature without defects; and $T^{0}(c)$ describes the critical transition temperature at defect concentration $c$. The coefficients in the Landau polynomial (normalized by the typical transformation "chemical" driving force $\left.\triangle f=1.85 \times 10^{6} \mathrm{~J} / \mathrm{m}^{3}[20]\right)$ are: $A_{1}^{0}=0.05 \cdot\left(T-T^{0}\right)$, $A_{2}=30, A_{3}=19$, and $A_{4}=10$.

The interfacial energy between the austenite and martensite is assumed to be $\gamma=0.05 \mathrm{~J} / \mathrm{m}^{2}$, which yields a length scale $l_{0}=0.94 \mathrm{~nm}$. The corresponding gradient energy coefficient is $\beta=4.5$. For an elastically isotropic system, the elastic modulus can be described through the shear modulus $G=40 \mathrm{GPa}$ and the Poisson ratio $\nu=0.3$. The elastic modulus is assumed to be the same for both phases (i.e., homogeneous modulus assumption). The elastic energy described in Equation (12) associated with the martensitic transformation or strain glass transition was described using the phase field microelasticity theory based on Green's function solution [21].

Microstructural evolution during martensitic and strain glass transitions is characterized by solving the stochastic time-dependent Ginsburg-Landau equation of the long range order parameters:

$$
\frac{d \eta_{p}(\mathbf{r}, t)}{d t}=-M \frac{\partial F}{\partial \eta_{p}(\mathbf{r}, t)}+\xi_{p}(\mathbf{r}, t), \quad p=1,2,
$$

where $M\left(M=1\right.$ in our simulation) is the kinetic coefficient and $\xi_{p}(\mathbf{r}, t)$ is the Langevin random noise term. The system size considered in the simulations is $256 l_{0} \times 256 l_{0}$ (i.e., $240 \mathrm{~nm} \times 240 \mathrm{~nm}$ ). Periodic boundary conditions were applied 
along both dimensions.

\subsection{Microstructure image analysis}

Each simulated image had a size of $256 \times 256$ pixels and was turned into a binary image by thresholding using a simple mean threshold algorithm [22]. Each binary image was then analyzed by scanning a window across the image, and the 2D second and fourth order moment invariants were computed for each window position. The pixels with binary value 1 make up the indicator function of the objects in the microstructure; all other pixels do not contribute to the moment integrals. Depending on the size of the scanning window, it is possible for one or more microstructural features to contribute to a single set of moment invariant values. The algorithm by Novotni and Klein was used for the numerical computation of the Cartesian moments [23]; this algorithm performs the two-fold integration analytically by separation of the integration variables and transforms the integral of equation 1 into a tensor product of quantities that can be computed by simple matrix multiplication, leading to an efficient numerical procedure in both $2 \mathrm{D}$ and 3D. An initial range of window sizes from $9 \times 9$ to $37 \times 37$ pixels was used to determine the optimal window size for the simulated microstructures. It was found that a window size of $17 \times 17$ pixels, which corresponds to representative elements with an area that is about $0.4 \%$ of the total image area, resulted in moment invariant maps with a sufficient number of data points as well as a clear evolution of the maps with respect to the relevant simulation parameters. The distribution of moment invariants is represented on both second order moment invariant maps (SOMIM) and projected moment invariant maps (PMIM). These density maps can be used to study how the MI distributions change with respect to temperature and defect concentration. 
The microstructure images were also characterized using a second image descriptor, the image entropy [24]. For a given image $A$, with gray levels in the range $[0 \ldots 255]$, an image histogram $h_{i}$ is obtained, which counts the number of pixels with gray level $i$. After normalization by the number of pixels, $N$, the im-

age entropy is computed from the values $p_{i}=h_{i} / N$ as $H(A)=-\sum_{i=0}^{255} p_{i} \ln p_{i}$. In thermodynamic terms, the image is regarded as an ideal solution of $256 \mathrm{com}-$ ponents, and the image entropy is equal to the configurational entropy of mixing of this ideal solution [25].

\section{Results and discussion}

\subsection{Moment invariant analysis}

Moment invariant density maps were generated for each condition in Table 1. Figures 3 to 5 show the SOMIM and PMIM for each microstructure and transformation type of Figure 1: austenite to martensite (Figure 3), austenite to premartensitic tweed to martensite (Figure 4), and austenite to strain glass (Figure 5). While the point defect concentration $c$ may slightly alter the starting microstructure, which is generated using identical noise terms (random number seed and noise amplitude), the density maps have similar moment invariant distributions, with mostly isotropic shapes dispersed through the structures, as shown in Figure 1. The premartensitic tweed and the strain glass microstructures look similar, and their density maps show similar microstructural shapes and shape distributions.

When $c=0$, the system transforms from its paraelastic parent phase to a polytwinned martensitic microstructure at a narrow temperature range (i.e., a sharp phase transition) that is typical of ferroelastics, as shown in Figure 1. Figure 3 
shows the density map of the austenite-martensite transformation. The starting microstructure exhibits a narrow range of moment invariants located on or near the isotropic shape parabola $\bar{\omega}_{2}=\bar{\omega}_{1}^{2}$. As the system cools down, the austenite transforms to martensite and displays a broader, more diffuse range of moment invariants, with a peak near the upper right corner $(1,1)$ point. The high moment invariant values are due to the poly-twinned nature of the martensite; since the interfaces between the twins are quite straight and the mask chosen to isolate the region of interest is a circle, the majority of moment invariant values will be close to those of the circle. Window positions that intersect the straight inter-variant boundaries give rise to the long curved distribution tails in Figure 3.

When $0<c<c_{c r i t}$, the parent phase first changes to premartensitic tweed before transforming into martensite at the martensite start temperature. The moment invariant density map evolution for this type of transformation is shown for $c=0.025$ in Figure 4. The parent phase with a low defect concentration has similar density maps to that of the undoped parent phase, with a mostly isotropic shape distribution. The premartensitic tweed exists at intermediate temperatures, which is characterized by a cross-hatched structure. This structure results in isotropic shapes that are distributed along the entirety of the isotropic shape parabola in the SOMIM. As the system cools, the distribution of moment invariants sharpens from the broad distribution at higher temperatures to one that becomes once again centered near $(1,1)$, similar to the $c=0$ case. The tweed microstructure changes into regular martensite upon further cooling because there are not enough point defects dispersed throughout the system to disrupt the long-range strain order; hence, the final microstructure at low defect concentrations is similar to that of the undoped system and exhibits martensite. While the density maps for the final temperature 
in $c=0$ and $c=0.025$ do not look the same, the maxima of both distributions are located near the upper right corner $(1,1)$, which are the moment invariants for the circle. Thus, the key identifying feature of the martensite microstructure is that its density maps peak near the point $(1,1)$ for the circular window used to determine the MIs.

For $c>c_{c r i t}$, the parent phase changes to a glass, similar to the premartensitic tweed, then to its final state as a strain glass with static nanodomains. As the microstructure cools from the parent phase, the density maps (Figure 5) do not change much and only move up slightly along the isotropic parabola. Since the initial structure becomes frozen as it cools, at the final temperature similar density maps are expected to those at the initial temperature. Thus, the strain glass state is characterized by density maps that are centered along the lower portion of the isotropic shape parabola, and the peak near $(1,1)$ never develops because the microstructure details remain much smaller than the circular window size used to determine the MIs.

The moment invariant density maps for the different microstructure states are clearly distinct and in principle allow for the microstructure types to be identified directly. The austenitic microstructure is characterized by a disordered strain state with MIs along the isotropic shape parabola in the range $[0.3,0.9]$, depending on the point defect concentration in the system. The martensite phase has a moment invariant distribution that is centered around $(1,1)$ due to the shape of the computational window (circular). The pre-martensitic tweed and the strain glass have density maps that are broadly similar to those of the parent phase, but vary in their details depending on the size of the nanodomains. As defect concentration increases, the domain size decreases, which shifts the moment invariant 
distributions. While the normal transformation has density maps that show different distributions for different temperatures, the density maps for systems with high defect concentrations do not change much as the system cools, and they are clearly distinguishable from those of the other microstructural states.

\subsection{Image entropy analysis}

The image entropy, $H$, at different defect concentrations $c$ and temperatures is shown in Figure 6. Results obtained for systems have defect concentrations below the critical value for strain glass are shown in different shades of blue, while those obtained for systems having defect concentrations above the critical values are shown in different shades of red. The defect concentrations are identified as having a martensitic transition or a strain glass transition based on the linearity of the relationship between entropy and temperature. A line of best fit is applied to each defect concentration, and an $\mathrm{R}^{2}$ value greater than 0.9 means that there is a nearly linear relationship between entropy and temperature for that defect concentration.

Low entropy images generally have little contrast between light and dark regions, whereas high entropy images tend to have a lot of texture and contrast. Hence, the strain glass microstructures will have a higher image entropy because of the strain disorder that is frozen into the system, while the martensitic microstructures will have a low image entropy due to the uniformity of the image contrast within the martensite variants. The parent phase has a relatively low image entropy at all defect concentrations since the microstructure does not change substantially with $c$; this is also supported by the moment invariant analysis.

For the undoped $(c=0)$ system, a rapid change occurs upon cooling from austenite to martensite with no intermediate state, so the image entropy rapidly 
changes from low to high during the transition and finally back to low for the uniform martensite variants. The highest image entropy occurs at $T=319.2 \mathrm{~K}$, likely because the martensitic plates are not fully developed at that temperature. The image entropy gradually decreases as the system cools down further.

The image entropy of systems with low defect concentrations starts out low, then increases quickly as the system cools. The entropy stays constant or decreases for the rest of the cooling process. The image entropy is at a peak at intermediate temperatures for systems with low defect concentrations (below 0.08). While the final microstructures are martensite, the interfaces between the two variants are not as sharply defined as in the undoped system, so they have higher entropy than in the undoped martensite. The higher the point defect concentration, the higher the image entropy of the final martensite microstructure.

Systems with high defect concentration start out with a low image entropy that steadily increases in nearly linear fashion upon cooling and can be fitted to a linear relationship. The formation of the martensitic nanodomains causes the entropy to increase, and so systems with more point defects will have a higher image entropy. However, the image entropy reaches a maximum and then decreases in systems with a low point defect concentration because the nanodomains in the precursory tweed structure transforms into micron scale martensitic twin plates. The image entropy displays a continuous change with temperature, which suggests a continuous transition. Whether or not one can determine an equivalent "image heat capacity" according to the image entropy is a topic of ongoing research.

Figure 7 shows the image entropy versus the defect concentration for selected temperatures. The entropy for the lowest temperature increases as defect concentration increases until the critical defect concentration, where the entropy is at a 
maximum. The entropy of the states with defect concentrations higher than the critical defect concentration decreases slightly from the maximum entropy found at $c=0.1$; however, these microstructures still have high entropy as compared to the entropy for low defect concentrations at $224 \mathrm{~K}$.

We conclude from these simulations that the strain glass state produces microstructures with a high image entropy, while the starting microstructure has low image entropy at all point defect concentrations. The premartensitic tweeds display a range of image entropies. Figure 6 shows that systems with low defect concentrations in the range $[0,0.08]$ have an entropy that displays no systematic relationship with respect to temperature. For higher concentrations, above 0.1 , the entropy follows a nearly linear relationship with temperature. The critical point defect concentration is thus determined to be $c=0.1$, when the system switches from undergoing a martensitic transition to a strain glass transition.

\section{Conclusions}

The strain glass transition in ferroelastics can be induced by doping with point defects. Different microstructural states can exist in a doped ferroelastic system, such as premartensitic tweed and a strain glass state, in addition to the typical austenite and martensite states. We have successfully used moment invariants and image entropy to automatically distinguish between these states. While the parent phase has a relatively narrow distribution of moment invariants, a system with low defect concentration as it is cooled displays a much greater spread in its moment invariant distribution. However, for a system with high defect concentration, the moment invariant distribution changes only by a small amount, indicating that it is indeed a frozen version of its former disordered strain state. 
The parent phase consists of equiaxed structural fluctuations randomly dispersed throughout the system. Its SOMIM has a small range of moment invariant values that lie near the isotropic shape parabola. The tweed structure has moment invariant maps that lie along the entirety of the parabola; due to the complex crosshatched state, when viewed through a small circular window, nearly all window positions reveal a close to isotropic intensity distribution, thus producing parabolic moment invariant maps. The strain glass state consists of nanosized martensitic domains that decrease in size as defect concentration increases. The strain glass has density maps similar to those of the parent phase, with a narrow distribution of moment invariants that lie along the isotropic shape parabola. The strain glass state can be distinguished from the premartensitic tweed because it has a smaller spread in moment invariant distribution.

Systems that undergo a martensitic transformation are characterized by a broad moment invariant distribution, with a peak centered near the circle point with coordinates $(1,1)$. However, systems that display a strain glass transition tend to have moment invariant distributions that do not change much upon cooling. These systems tend to have more complex shapes in their strain domain microstructure, giving rise to MI distributions away from the circle point. Since the calculated moment invariants all lie near the isotropic shape parabola $\bar{\omega}_{2}=\bar{\omega}_{1}^{2}$, the shapes detected by the moment invariant windowing technique are nearly isotropic.

In terms of image entropy, we find that both the parent phase and the final martensitic phase display low image entropy whereas the premartensitic tweed state has an intermediate image entropy. The strain glass state displays varying entropy values as well, but systems with high defect concentrations have higher entropy values than systems with low defect concentrations because of how the 
nanodomains finally rearrange in the microstructure. Systems with defect concentrations equal or greater than $c_{c r i t}=0.1$ display an image entropy that decreases nearly linearly with temperature, or, equivalently, increases linearly with time.

Moment invariants have been shown to be capable of characterizing the range of strain domain shapes that can occur in the different microstructural states of a ferroelastic system. Image entropy can be used to determine objectively what state the system is in and to identify the critical defect concentration, which, for the computational model employed in this paper, is $c_{\text {crit }}=0.1$ and is in agreement with the value determined independently by heat capacity calculations in [11].

\section{Acknowledgements}

LN and MDG would like to acknowledge the Air Force Office of Scientific Research, FA-95501210475, for financial support. DW would like to acknowledge National Basic Research Program of China (2012CB619402, 2014CB 644003), National Natural Science Foundation of China (511201125); YW would like to acknowledge the US National Science Foundation DMR-1410322 and US Department of Energy Grant No. DE-SC0001258. 
[1] Otsuka K, Wayman CM. Shape memory materials. New York: Cambridge University Press; 1998.

[2] Otsuka K, Ren X. Physical metallurgy of Ti-Ni-based shape memory alloys. Prog Mat Sci 2005;50:511-678.

[3] Sarkar S, Ren X, Otsuka K. Evidence for strain glass in the ferroelasticmartensitic system $\mathrm{Ti}_{50-x} \mathrm{Ni}_{50+x}$. Phys Rev Lett 2005;95:205702.

[4] Wang D, Zhang Z, Zhang J, Zhou Y, Wang Y, Ding X, Wang Y, Ren X. Strain glass in Fe-doped Ti-Ni. Acta Mater 2010;58:6206-6215.

[5] Zhou Y, Xue D, Ding X, Wang Y, Zhang J, Zhang Z, Wang D, Otsuka K, Sun J, Ren X. Strain glass in doped $\mathrm{Ti}_{50}\left(\mathrm{Ni}_{50 x} \mathrm{D}_{x}\right)(\mathrm{D}=\mathrm{Co}, \mathrm{Cr}, \mathrm{Mn})$. Acta Mater 2010;58:5433-5442.

[6] Lloveras P, Castán T, Porta M, Planes A, Saxena A. Influence of elastic anisotropy on structural nanoscale textures. Phys Rev Lett 2008;10:165707.

[7] Vasseur R, Lookman T. Effects of disorder in ferroelastics: A spin model for strain glass. Phys Rev B 2010;81:094107.

[8] Wang D, Wang Y, Zhang Z, Ren X. Modeling abnormal strain states in ferroelastic systems: the role of point defects. Phys Rev Lett 2010;105:205702.

[9] Ren X, Wang Y, Zhou Y. Strain glass in ferroelastic systems: premartensitic tweed versus strain glass. Philos Mag 2010;90:141-157.

[10] Wang D, Hou S, Wang Y, Ding X, Ren S, Ren X, Wang Y. Superelasticity of slim hysteresis over a wide temperature range by nanodomains of martensite. Acta Mater 2014;66:349-359. 
[11] Wang D, Ni Y, Gao J, Zhang Z, Ren X, Wang Y. Unique properties associated with normal martensitic transition and strain glass transition - a simulation study. J Alloys Comp 2013;577S:S102-S106.

[12] Wang Y, Ren X, Otsuka K, Saxena A. Evidence for broken ergodicity in strain glass. Phys Rev B 2007;76:132201.

[13] Zhou Y, Xue D, Ding X, Otsuka K, Sun J, Ren X. High temperature strain glass in $\mathrm{Ti}_{50}\left(\mathrm{Pd}_{50-x} \mathrm{Cr}_{x}\right)$ alloy and shape memory effect and superelasticity. Appl Phys Lett 209;95:151906.

[14] Zhang Z, Wang Y, Wang D, Zhou Y, Otsuka K, Ren X. Phase diagram of $\mathrm{Ti}_{50-x} \mathrm{Ni}_{50+x}$ : crossover from martensite to strain glass. Phys Rev B 2010;81:224102.

[15] Hu MK. Visual pattern recognition by moment invariants. IEEE Trans Inf Theory 1962;8:179-187.

[16] MacSleyne JP, Simmons JP, De Graef M. On the use of 2-D moment invariants for the automated classification of particle shapes. Acta Mater 2008;56:427-437.

[17] Callahan PG, Simmons JP, De Graef M. A quantitative description of the morphological aspects of materials structures suitable for quantitative comparison of 3D microstructures. Mod Sim Mat Sci Eng 2013;21:015003.

[18] Semenovskaya S, Khachaturyan AG. Coherent structural transformations in random crystalline systems. Acta Mater 1997;45:4367-4384. 
[19] Levaniuk AP, Sigov AS. Defects and structural phase transitions. New York: Gorden and Breach Science Publishers; 1988.

[20] Eggeler G, Khalil-Afllafi J, Gollerthan S, Somsen C, Schmahl W, Sheptyakov D. On the effect of aging on martensitic transformations in Ni-rich NiTi shape memory alloys. Smart Mater Struct 2005;14:S186-S191.

[21] Khachaturyan AG. Theory of Structural Transformations in Solids. New York: John Wiley and Sons Ltd.; 1983.

[22] Szeliski R. Computer Vision: Algorithms and Applications. London: Springer; 2010.

[23] Novotni M, Klein R. Shape retrieval using 3D Zernike descriptors. Computer Aided Design 2004;36:1047-1062.

[24] Cover TM, Thomas JA. Elements of Information Theory. New York: Wiley Interscience; 1991.

[25] Gulsoy EB, Simmons JP, De Graef M. Application of joint histogram and mutual information to registration and data fusion problems in serial sectioning microstructure studies. Scr Mater 2009;60:381-384. 
Table 1: Defect Concentration and Temperature $(\mathrm{K})$ for five different simulation sets.

\begin{tabular}{|c|c|c|c|c|c|}
\hline Concentration & Temp 1 & Temp 2 & Temp 3 & Temp 4 & Temp 5 \\
\hline 0.0 & - & 322 & 319.2 & 274.4 & 224 \\
0.01 & 330.4 & 322 & 308 & 252 & 224 \\
0.025 & 324.8 & 322 & 319.2 & 282.8 & 224 \\
0.03 & 330.4 & 322 & 308 & 252 & 224 \\
0.05 & 322 & 319.2 & 316.4 & 308 & 224 \\
0.06 & 330.4 & 322 & 308 & 252 & 224 \\
0.07 & 322 & 316.4 & 308 & 280 & 224 \\
0.08 & 322 & 316.4 & 308 & 280 & 224 \\
0.1 & 316.4 & 308 & 294 & 277.2 & 224 \\
0.125 & 313.6 & 308 & 294 & 280 & 224 \\
0.15 & 313.6 & 308 & 288.4 & 263.2 & 224 \\
0.2 & 313.6 & 308 & 288.4 & 268.8 & 224 \\
\hline
\end{tabular}




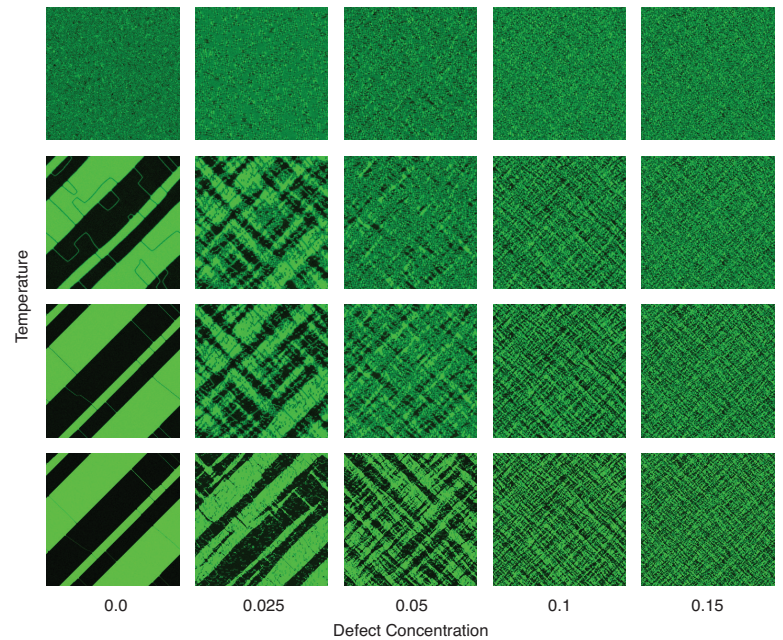

Figure 1: Microstructures resulting from the simulation with various defect concentrations. The temperature decreases from top to bottom. 


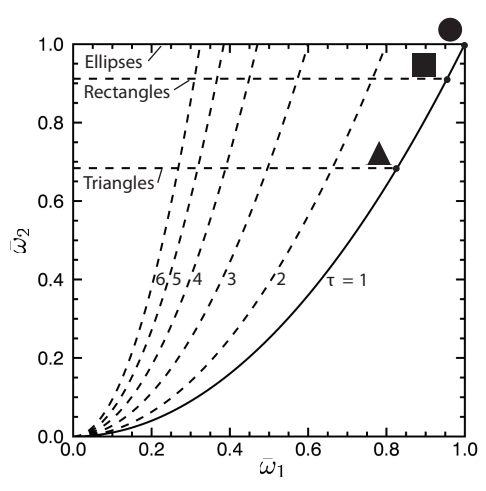

(a) Second order moment invariant map (SOMIM)

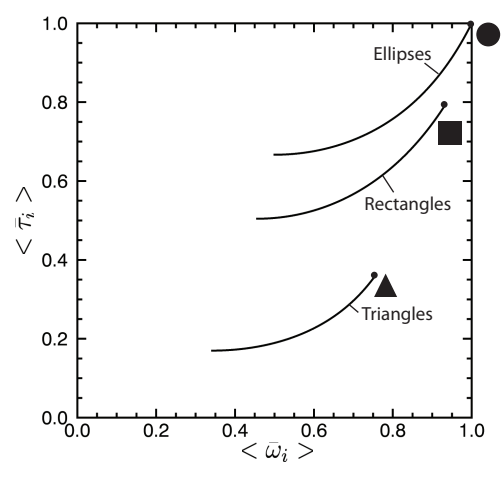

(b) Projected moment invariant map (PMIM)

Figure 2: The Second-Order Moment Invariant Map is shown on the left; all 2D shapes are contained within the region $0 \leq \bar{\omega}_{1}^{2} \leq \bar{\omega}_{2} \leq 1$; all 2D shapes correspond to points to the left of the parabola $\bar{\omega}_{2}=\bar{\omega}_{1}^{2}$. The Projected-Moment Invariant Map is shown on the right, along with the locations of important shapes and their affine transformations (curved lines, corresponding to the horizontal dashed lines in the SOMIM). 

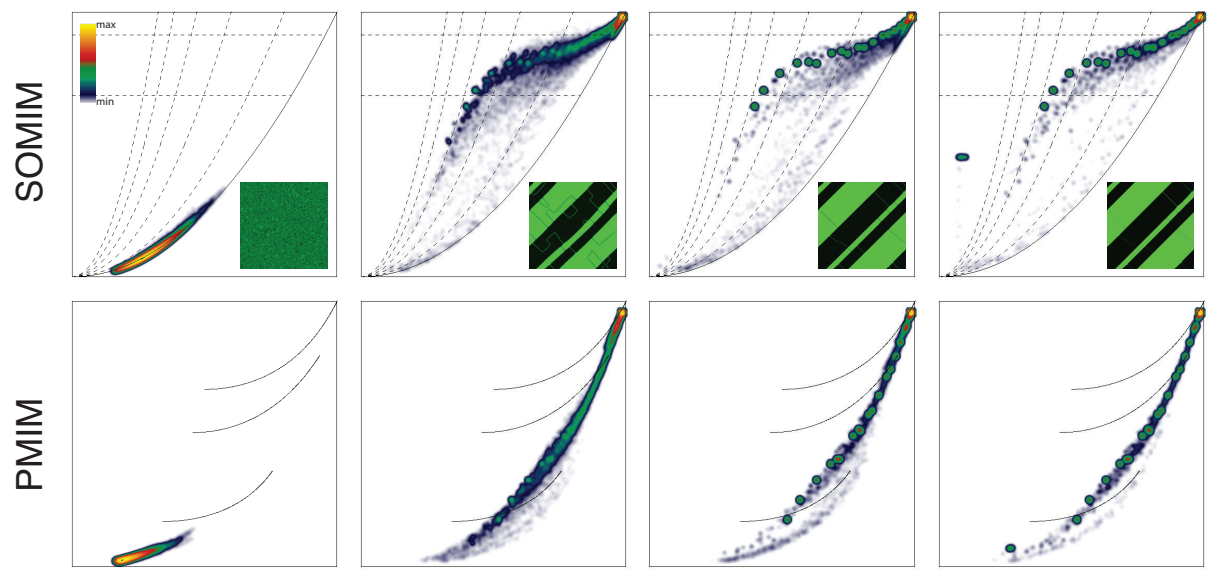

322

319.2

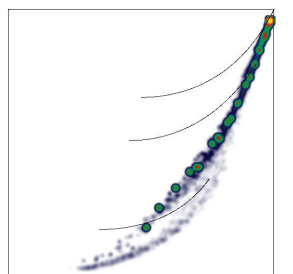

274.4

Temperature, $\mathrm{T}(\mathrm{K})$

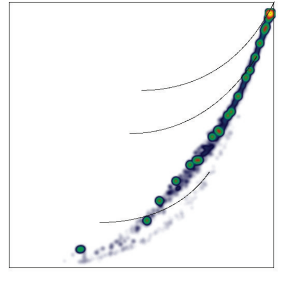

263.2

Figure 3: The SOMIM and PMIM density maps of the system with a defect concentration of $c=0$, with decreasing temperature. A transformation from the parent phase $(\mathrm{T}=322)$ to martensite $(\mathrm{T}=319.2,274.4,263.2)$ with no intermediate states is shown. 

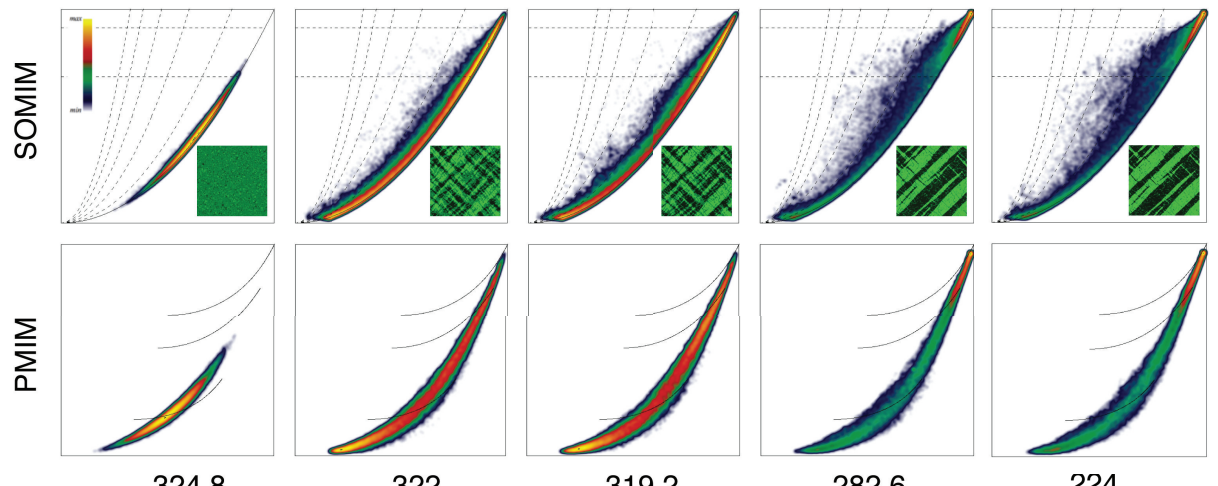

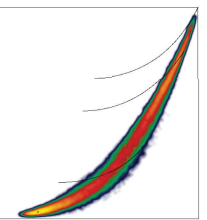

319.2

Temperature

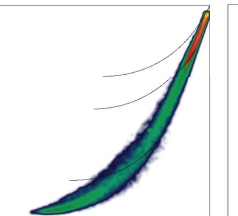

282.6

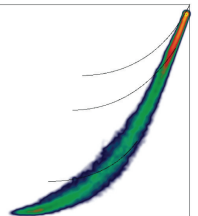

224

Figure 4: The SOMIM and PMIM density maps of the system with a defect concentration of $c=0.025$, with decreasing temperature (in $\mathrm{K}$ ). This shows a transformation from the parent phase $(\mathrm{T}=324.8 \mathrm{~K})$ to the premartensitic tweed $(\mathrm{T}=322,319.2 \mathrm{~K})$ to martensite $(\mathrm{T}=224 \mathrm{~K})$. 


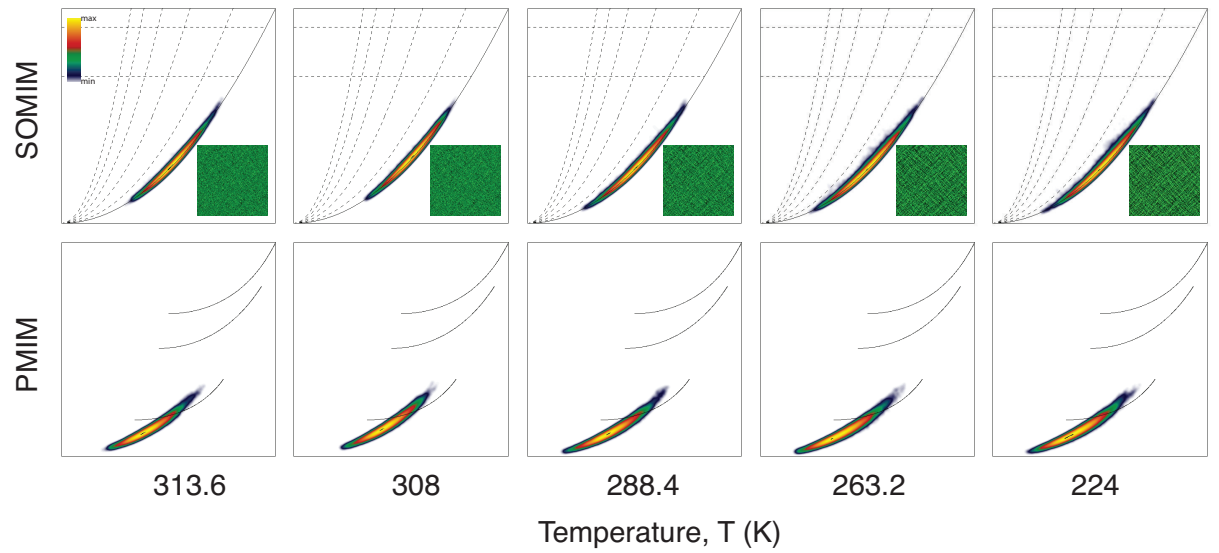

Figure 5: SOMIM and PMIM density maps for the system with a defect concentration of $c=0.15$, with decreasing temperature. This shows a strain glass transition instead of the martensitic transformation, evolving from the parent phase $(\mathrm{T}=313.6 \mathrm{~K})$ to the frozen strain glass state $(\mathrm{T}=308 \mathrm{~K}$, $288.4,263.2,224 \mathrm{~K})$. 


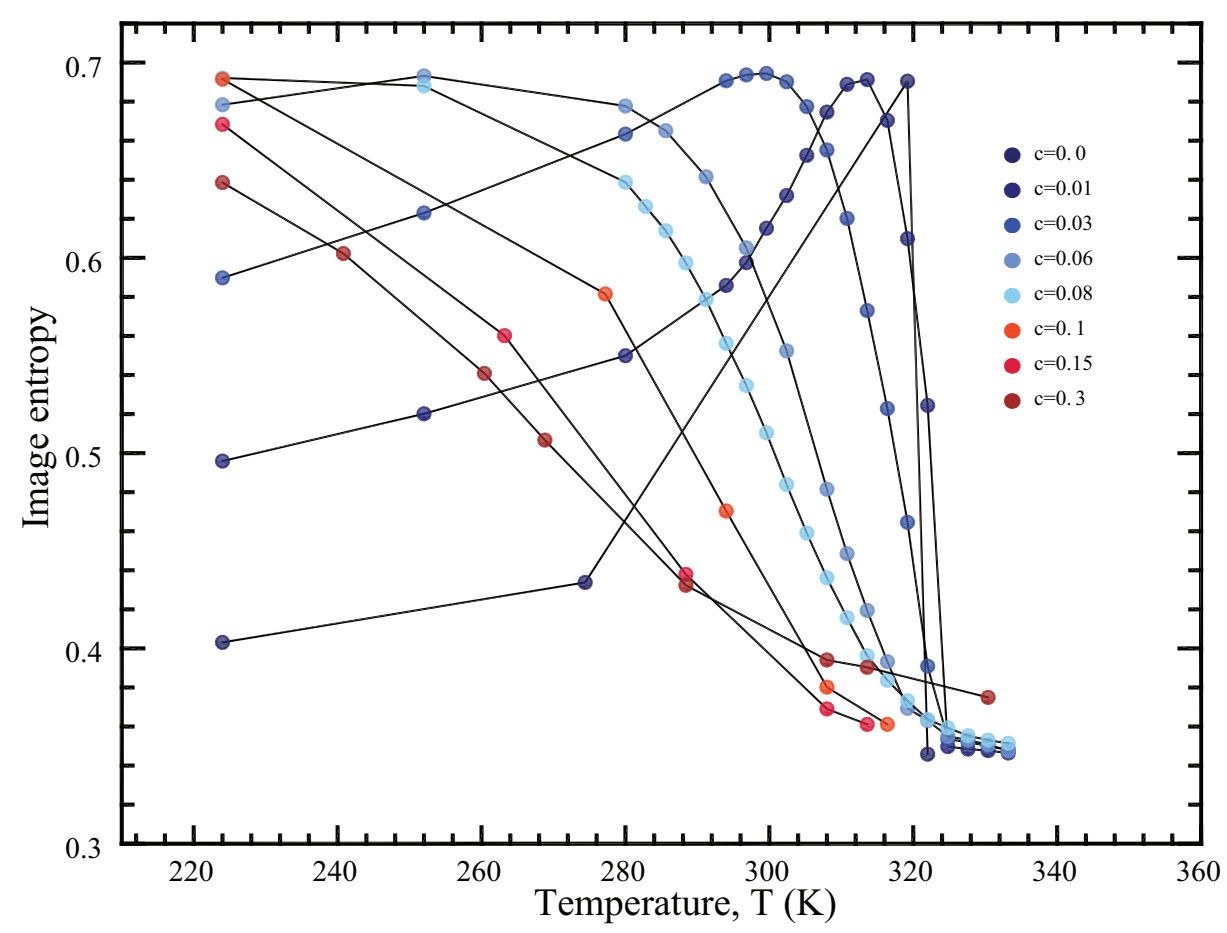

Figure 6: Image entropy vs. temperature for varying defect concentrations. Note the shift towards the left, from a strongly peaked curve to a decreasing, nearly linear relationship for systems above the critical defect concentration. 


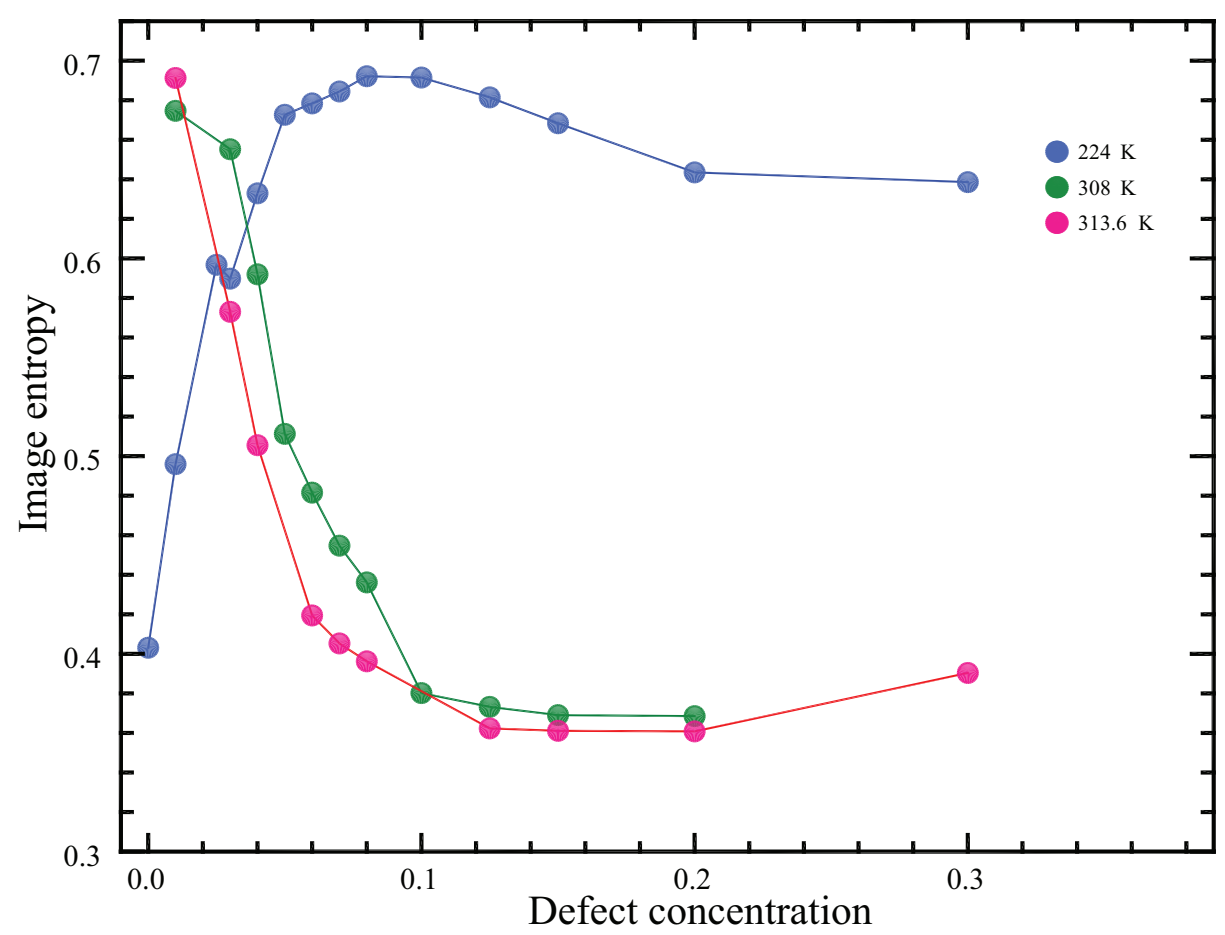

Figure 7: Entropy vs. defect concentration for different simulation temperatures. At intermediate temperatures, the entropy decreases as the defect concentration increases. At the final temperature (224K), the entropy increases with increasing defect concentration until it reaches its maximum at about $c=0.1$. 